\title{
A Review on Chemical Constituents and Biological Activities of the Genus Beilschmiedia (Lauraceae)
}

\author{
Wan Mohd Nuzul Hakimi Wan Salleh, Farediah Ahmad ${ }^{1 *}$, Khong Heng Yen ${ }^{2}$ \\ and Razauden Mohamed Zulkifli ${ }^{3}$ \\ ${ }^{1}$ Department of Chemistry, Faculty of Science, Universiti Teknologi Malaysia, Skudai, 81310 Johor Bahru, Johor, ${ }^{2}$ School of \\ Chemistry and Environmental Studies, Faculty of Applied Sciences, Universiti Teknologi MARA Sarawak, Kota Samarahan, \\ 94300, Sarawak, ${ }^{3}$ Department of Bioscience and Health Sciences, Faculty of Biosciences and Medical Engineering, Universiti \\ Teknologi Malaysia (UTM), 81310 Skudai, Johor, Malaysia
}

${ }^{*}$ For correspondence: Email: farediah@kimia.fs.utm.my; Tel: +607-5534137; Fax: +607-5566162

\begin{abstract}
The current review is aimed to deliver some updates on the ethnobotany, phytochemistry and biological activities of Beilschmiedia species in order to throw more light on their therapeutic potentials and future research priorities. Phytochemical studies on Beilschmiedia genus yielded essential oils, endiandric acid derivatives, amides/alkaloids, lignans/neoligans, flavonoids and miscellaneous compounds, while bioactivity studies revealed that the extracts and compounds displayed a broad spectrum of biological activities. Only 24 species of the genus Beilschmiedia have been studied phytochemically thus far. Therefore, there is a need to ascertain the pharmacological properties of the plant extracts and new compounds from this genus. More studies on the standardization and pharmacology of Beilschmiedia extracts need to be conducted to ascertain new pharmacological properties and their biochemical and physiological mechanisms. Furthermore, detailed preclinical toxicity, bioavailability, pharmacokinetics and pharmacodynamics of extracts of the species and their isolated compounds require additional investigation in order to verify their safety and determine their suitability as sources of some current medicines.
\end{abstract}

Keywords: Beilschmiedia, Lauraceae, ethnobotany, phytochemistry, pharmacology

\begin{abstract}
Tropical Journal of Pharmaceutical Research is indexed by Science Citation Index (SciSearch), Scopus, International Pharmaceutical Abstract, Chemical Abstracts, Embase, Index Copernicus, EBSCO, African Index Medicus, JournalSeek, Journal Citation Reports/Science Edition, Directory of Open Access Journals
\end{abstract} (DOAJ), African Journal Online, Bioline International, Open-J-Gate and Pharmacy Abstracts

\section{INTRODUCTION}

The genus Beilschmiedia is a pantropical genus of about 287 species mainly in Southeast Asia and Africa. About 81 Beilschmiedia species have been identified in inter-tropical Africa with 41 species in Cameroon alone [1,2]. Plants of the genus Beilschmiedia have been listed in the plant list (2013). 287 of the genus names are accepted and the remaining names are either synonym or unresolved names. Revisional studies of the genus have been made in several regions, such as China, Madagascar, Australia and the Neotropics. In recent years, Kostermans described many new taxa of Beilschmiedia from Malay Peninsular and Southern Thailand [3-9]. Kochummen also revised Lauraceae and published in Tree Flora of Malaya [10]. Van der Werff studied the genera of Lauraceae in the Flora Malesiana Region and two reports on the synopsis of genus Beilschmiedia in Madagascar have been published $[11,12]$.

The ethnopharmacological history of Beilschmiedia indicates that some species have been used in local medicine to treat various 
conditions, including uterine tumors, rheumatism, pulmonary disorders, bacterial infections, malaria and tuberculosis [13-17]. Because of their versatile therapeutic traditional uses, extensive phytochemical investigations have been carried out on Beilschmiedia plants which led to isolation of numerous classes of secondary metabolites such as terpenoids, endiandric acid derivatives, essential oils, fatty acids, lignans/neolignans, flavonoids, amide/alkaloids and other components. Endiandric acid derivatives are considered to be the main constituents in Beilschmiedia species and are responsible for many of their biological and pharmacological activities [18-22].

Both in vivo and in vitro experiments have indicated that the Beilschmiedia plant extracts possess a variety of pharmacological aspects with antioxidant, anti-inflammatory, cytotoxicity, antibacterial and antifungal activities [23-26]. With consideration of the importance of the therapeutic property of this genus to heal various ailments. Therefore a broader range of studies on the bioactivities need to be performed to ascertain the pharmacological properties. These gaps lead to systematic research activities on phytochemicals and pharmacology of genus Beilschmiedia. As extensive studies on Beilschmiedia species have not been conducted, there are thus significant opportunities to discover novel bioactive compounds and beneficial medicinal and pharmacological properties from various extracts of the species are warranted. The available information on various species belonging to the genus Beilschmiedia were collected via electronic search (using Pubmed, SciFinder, Scopus, Google Scholar, JCCC@INSTIRC and Web of Science) and a library search for articles published in peer-reviewed journals.

\section{DISTRIBUTION, BOTANICAL DESCRIPTION AND ETHNOBOTANY}

The genus Beilschmiedia comprises more of trees and rarely shrubs, and is usually distinguished from other genera of the Lauraceae by the following characteristics: paniculate or racemose inflorescences that are not strictly cymose at the terminal division, bisexual and trimerous flowers with six equal to subequal tepals, six to nine fertile stamens representing the outer two or three whorls, two-celled anthers, and fruits lacking cupules. This family is also recognized by the simple, alternate, stiff and aromatic elliptic to obovate leaves, and by the fruits often borne in a cup [27]. It is placed in the tribe Cryptocaryeae Nees together with other core genera such as Cryptocarya, Endiandra and Potameia based on the wood and bark anatomy and inflorescence structure [27]. Most of the species grow in tropical climates but few of them are native to the temperate regions. They are widespread in tropical Asia, Africa, Australia, New Zealand, Central America, Caribbean and South America [28]. In the Southeast Asia, the genus can be found in Myanmar, Vietnam, Cambodia, Thailand, Malaysia, Philippines, Indonesia and various islands, such as Sumatra and Java [29]. Beilschmiedia is categorized as a genus of forest trees, predominantly from low to high elevation. B. glauca is found in the upper evergreen forest and rarely found in the lowland evergreen forest, $B$. maingayi is found in the upper evergreen forest, over 1,200 m altitude, $B$. membranacea is found in the montane evergreen forest and $B$. palembanica is found in the lower evergreen forest to the upper evergreen forest. Many species of Beilschmiedia were reported in Bhutan and Sikkim, such as B. gammieana in montane forest with oaks, and broad leaves forest at 1,750-2,050 m altitude, B. assamica in subtropical forest and broad leaves forest and in temperate zone at 300-1,800 m altitude and $B$. roxburghiana in subtropical forest at $200-400 \mathrm{~m}$ altitude [30,31].

Available literature and information show that several Beilschmiedia species have been applied as traditional medicines in various parts of the world. This genus has an economic importance in forest products for use as timber and herbal medicine. The most prevalent usages are for treating bone related problems which include arthritis, rheumatism and renal ailments. The important ethnomedicinal properties possessed by this genus can be the basis for further research to determine the phytochemical and pharmacological aspects of the above mentioned genus. The lists of the various medicinal uses of Beilschmiedia species in different parts of the world are shown in Table 1.

\section{CHEMICAL CONSTITUENTS}

Systematic phytochemical investigation is important to understand the pharmacology of the species as well as the mechanisms of action and for quality control purposes. The phytochemical studies of various species of the genus have successfully afforded numerous chemical components. They were essential oils, endiandric acid derivatives, amides and alkaloids, lignans and neolignans, flavonoids and also miscellaneous compounds (cyanogenic glycosides, benzopyran, benzenoid, terpenes, benzaldehyde, and fatty acid). The major 
components of the essential oil compositions from several species of Beilschmiedia species are tabulated in Table 2. The phytochemical studies of Beilschmiedia species are shown in Table 3.

Table 1: Medicinal uses of several Beilschmiedia species

\begin{tabular}{|c|c|c|c|c|}
\hline Species & Local name & Locality & Plant parts and medicinal uses & Ref \\
\hline B. gaboonensis & Kanda & Cameroon & $\begin{array}{l}\text { Fruits: as appetite stimulants and spices } \\
\text { Bark: analgesic and healing ointments }\end{array}$ & [13] \\
\hline B. manii & Bubueh sapo & Cameroon & $\begin{array}{l}\text { Fruits: as appetite stimulants, spices and } \\
\text { treatment of dysentery and headache }\end{array}$ & \\
\hline B. zenkeri & Not reported & Cameroon & Fruits: as appetite stimulants and spices & \\
\hline B. cryptocaryoides & Not reported & Madagascar & Fruits/bark: to treat infectious diseases & [21] \\
\hline B. acuta & Ndareh & Cameroon & $\begin{array}{c}\text { Leaf: cancer and gastrointestinal } \\
\text { infections }\end{array}$ & [24] \\
\hline B. obscura & Not reported & Cameroon & Bark: gastrointestinal infection & [25] \\
\hline B. sphaerocarpa & Not reported & Indochina & $\begin{array}{l}\text { Bark :herbs to cure skin related disease } \\
\text { such as scabies, acne and pustule }\end{array}$ & [32] \\
\hline B. pahangensis & Not reported & Malaysia & $\begin{array}{c}\text { Bark: as a drink after childbirth and also } \\
\text { to assuage stomach ache, diarrhea and } \\
\text { dysentery }\end{array}$ & [33] \\
\hline B. tonkinensis & Perapoh & Malaysia & $\begin{array}{l}\text { Leaf: to make medicine for easing pain, } \\
\text { inflammation and broken bone }\end{array}$ & \\
\hline B. madang & Medang merah & Indonesia & $\begin{array}{c}\text { Wood: the decoction as an antimalarial } \\
\text { preparation }\end{array}$ & [34] \\
\hline B. anacardioides & Ntseum & Cameroon & $\begin{array}{l}\text { Stem bark: to cure uterine tumors, } \\
\text { rubella, female genital infections, and } \\
\text { rheumatisms }\end{array}$ & [56] \\
\hline B. lancilimba & Not reported & Cameroon & Bark: to cure skin bacterial infections & [57] \\
\hline B. podagrica & Kunana & New Guinea & Leaf/bark: treatment of stomach ailments & [58] \\
\hline B. tawa & Tawa & New Zealand & $\begin{array}{l}\text { Bark: stomachaches, colds and decoction } \\
\text { for wounds }\end{array}$ & [59] \\
\hline
\end{tabular}

Table 2: Major components of the essential oils of Beilschmiedia species

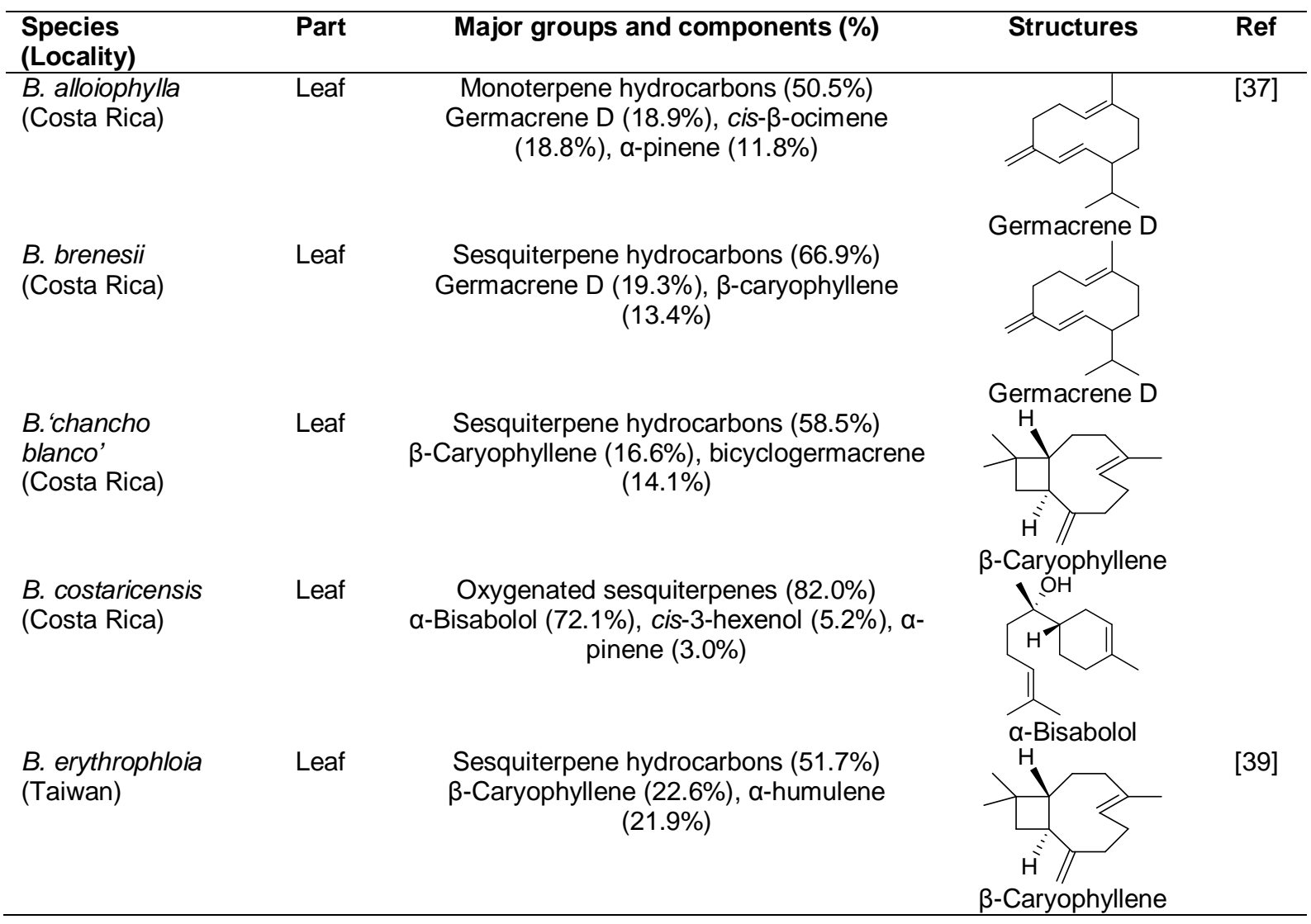


Table 2: Major components of the essential oils of Beilschmiedia species (continued)

\begin{tabular}{|c|c|c|c|c|}
\hline $\begin{array}{l}\text { Species } \\
\text { (Locality) }\end{array}$ & Part & Major groups and components (\%) & Structures & Ref \\
\hline $\begin{array}{l}\text { B. madang } \\
\text { (Malaysia) }\end{array}$ & Bark & $\begin{array}{c}\text { Sesquiterpene hydrocarbons }(63.8 \%) \\
\delta \text {-Cadinene (17.0\%), } \alpha \text {-cubebene }(11.3 \%), \beta- \\
\text { caryophyllene }(10.3 \%) \\
\text { Sesquiterpene hydrocarbons }(65.3 \%) \\
\delta \text {-Cadinene }(20.5 \%) \text {, } \alpha \text {-cubebene }(15.6 \%), \alpha- \\
\text { cadinol }(10.6 \%)\end{array}$ & & [40] \\
\hline \multirow[t]{2}{*}{$\begin{array}{l}\text { B. miersii } \\
\text { (United States/ } \\
\text { Chile) }\end{array}$} & Leaf & $\begin{array}{c}\text { Not mention } \\
\text { Germacrene D (24.8\%), } \alpha \text {-terpinene (10.0\%), } \\
\text { Y-curcumene (9.6\%) }\end{array}$ & & [36] \\
\hline & Leaf & $\begin{array}{l}\text { Not mention } \\
\text { Sarisan, myristicin, elemicin, asarone }\end{array}$ & & [35] \\
\hline \multirow[t]{2}{*}{$\begin{array}{l}\text { B. pendula } \\
\text { (Costa Rica) }\end{array}$} & Leaf & $\begin{array}{c}\text { Sesquiterpene hydrocarbons (33.0\%) } \\
\beta \text {-Pinene (10.4\%), } \beta \text {-caryophyllene (8.6\%), } \\
\alpha \text {-pinene }(7.9 \%)\end{array}$ & & [38] \\
\hline & Branch & $\begin{array}{c}\text { Sesquiterpene hydrocarbons (59.3\%) } \\
\beta \text {-Caryophyllene (17.3\%), } \beta \text {-selinene }(9.1 \%) \text {, } \\
\text { bicyclogermacrene }(8.9 \%)\end{array}$ & & \\
\hline $\begin{array}{l}\text { B. tarairie } \\
\text { (United States) }\end{array}$ & Leaf & $\begin{array}{c}\text { Not mention } \\
\alpha-P \text { inene }(17.8 \%), \beta-\text { pinene }(9.4 \%), \\
\text { germacrene D (6.6\%) }\end{array}$ & & [36] \\
\hline $\begin{array}{l}\text { B. tilaranensis } \\
\text { (Costa Rica) }\end{array}$ & Leaf & $\begin{array}{c}\text { Sesquiterpene hydrocarbons (85.1\%) } \\
\text { Germacrene D (54.9\%), } \beta \text {-caryophyllene } \\
(14.8 \%)\end{array}$ & & [37] \\
\hline $\begin{array}{l}\text { B. pulverulenta } \\
\text { (Malaysia) }\end{array}$ & $\begin{array}{l}\text { Leaf/ } \\
\text { Bark }\end{array}$ & $\begin{array}{c}\text { Phenylpropanoids (51.1\%) } \\
\text { Eugenol (45.3\%) }\end{array}$ & & [41] \\
\hline
\end{tabular}

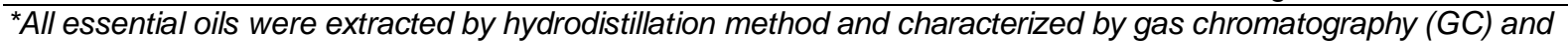
gas chromatography-mass spectrometry (GC-MS)

Table 3: Chemical constituents isolated from Beilschmiedia species

\begin{tabular}{llll}
\hline Compound & Species & Plant part & Ref \\
\hline Endiandric acid derivatives & & & \\
\hline Beilcyclone A & B. erythrophloia & Roots & {$[18]$} \\
Beilschmiedic acid A & B. anacardioides & Stem bark & {$[44,46]$} \\
& Beilschmiedia sp. & Leaves & {$[20]$} \\
Beilschmiedic acid B & B. anacardioides & Stem bark & {$[44]$} \\
Beilschmiedic acid C & B. anacardioides & Lem bark & {$[44,46]$} \\
& Beilschmiedia sp. & Stem bark & {$[20]$} \\
Beilschmiedic acid D & B. anacardioides & Stem bark & {$[17]$} \\
Beilschmiedic acid E & B. anacardioides & Stem bark & {$[46]$} \\
Beilschmiedic acid F & B. anacardioides & Stem bark & {$[46]$} \\
Beilschmiedic acid G & B. anacardioides & Leaves & {$[20]$} \\
Beilschmiedic acid H & Beilschmiedia sp. & & \\
\hline
\end{tabular}


Table 3: Chemical constituents isolated from Beilschmiedia species (continued)

\begin{tabular}{|c|c|c|c|}
\hline Compound & Species & Plant part & Ref \\
\hline Beilschmiedic acid I & Beilschmiedia sp. & Leaves & \\
\hline Beilschmiedic acid J & Beilschmiedia sp. & Leaves & \\
\hline Beilschmiedic acid K & Beilschmiedia sp. & Leaves & \\
\hline Beilschmiedic acid L & Beilschmiedia sp. & Leaves & \\
\hline Beilschmiedic acid M & Beilschmiedia sp. & Leaves & \\
\hline Beilschmiedic acid N & Beilschmiedia sp. & Leaves & \\
\hline Beilschmiedic acid O & Beilschmiedia sp. & Leaves & \\
\hline Beilschmiedin & B. anacardioides & Stem bark & [17] \\
\hline Cryptobeilic acid A & B. cryptocaryoides & Bark & [21] \\
\hline Cryptobeilic acid B & B. cryptocaryoides & Bark & \\
\hline Cryptobeilic acid C & B. cryptocaryoides & Bark & \\
\hline Cryptobeilic acid D & B. cryptocaryoides & Bark & \\
\hline Endiandric acid A & B. obtusifolia & Leaves & [14] \\
\hline Endiandric acid B & B. tooram & Leaves & \\
\hline Endiandric acid C & B. tooram & Leaves & \\
\hline Endiandric acid $\mathrm{H}$ & B. fulva & Stem & [42] \\
\hline Endiandric acid I & B. erythrophloia & Roots & [43] \\
\hline Endiandric acid $\mathrm{J}$ & B. erythrophloia & Roots & \\
\hline Endiandric acid $\mathrm{K}$ & B. tsangii & Roots & [19] \\
\hline Endiandric acid L & B. tsangii & Roots & \\
\hline Endiandric acid M & B. tsangii & Roots & [45] \\
\hline Endiandramide A & B. tsangii & Roots & [19] \\
\hline Endiandramide B & B. tsangii & Roots & \\
\hline Erythrophloin A & B. erythrophloia & Roots & [18] \\
\hline Erythrophloin B & B. erythrophloia & Roots & \\
\hline Erythrophloin C & B. erythrophloia & Roots & \\
\hline Erythrophloin D & B. erythrophloia & Roots & \\
\hline Erythrophloin E & B. erythrophloia & Roots & \\
\hline Erythrophloin F & B. erythrophloia & Roots & \\
\hline Ferrugineic acid $A$ & B. ferruginea & Leaves/flower & [22] \\
\hline Ferrugineic acid B & B. ferruginea & Leaves/flower & \\
\hline Ferrugineic acid $\mathrm{C}$ & B. ferruginea & Leaves/flower & \\
\hline Ferrugineic acid D & B. ferruginea & Leaves/flower & \\
\hline Ferrugineic acid $\mathrm{E}$ & B. ferruginea & Leaves/flower & \\
\hline Ferrugineic acid F & B. ferruginea & Leaves/flower & \\
\hline Ferrugineic acid $\mathrm{G}$ & B. ferruginea & Leaves/flower & \\
\hline Ferrugineic acid $\mathrm{H}$ & B. ferruginea & Leaves/flower & \\
\hline Ferrugineic acid I & B. ferruginea & Leaves/flower & \\
\hline Ferrugineic acid $\mathrm{J}$ & B. ferruginea & Leaves/flower & \\
\hline Ferrugineic acid $\mathrm{K}$ & B. ferruginea & Leaves/flower & \\
\hline Tsangibeilin A & B. tsangii & Roots & [19] \\
\hline \multirow[t]{2}{*}{ Tsangibeilin B } & B. tsangii & Roots & \\
\hline & B. cryptocaryoides & Bark & [21] \\
\hline Tsangibeilin C & B. tsangii & Roots & {$[45]$} \\
\hline Tsangibeilin D & B. tsangii & Roots & \\
\hline Tricyclotsangibeilin & B. tsangii & Roots & \\
\hline Armepavine & B. brevipes & Leaves & [48] \\
\hline Asimilobine & B. alloiophylla & Bark & [49] \\
\hline \multirow[t]{2}{*}{ (+)-Boldine } & B. kunstleri & Bark & [47] \\
\hline & B. alloiophylla & Bark & [49] \\
\hline (+)-Cassythicine & B. kunstleri & Leaves/bark & {$[47,51]$} \\
\hline (+)-Dehatrine & B. madang & Wood & [34] \\
\hline$O, O$-Dimethylannocherin A & B. brevipes & Leaves & [48] \\
\hline 0,0 -Dimethylcoclaurine & B. brevipes & Leaves & \\
\hline (+)-N-Dimethylphyllocaryptine & B. kunstleri & Bark & {$[47,49]$} \\
\hline $\begin{array}{l}\text { (6,7-Dimethoxyisoquinolinyl)-(4'-methoxyphenyl)- } \\
\text { methanone }\end{array}$ & B. brevipes & Leaves & {$[48]$} \\
\hline $\begin{array}{l}\text { (6,7-Dimethoxy-4-methylisoquinolinyl)-(4'- } \\
\text { methoxyphenyl)-methanone }\end{array}$ & B. brevipes & Leaves & \\
\hline Dioxamine & B. erythrophloia & Roots & [18] \\
\hline Glaucine & B. podagrica & Leaves & [60] \\
\hline 2-Hydroxy-9-methoxyaporphine & B. alloiophylla & Bark & [49] \\
\hline (+)-2-Hydroxy-1,9,10-trimethoxyaporphine & B. podagrica & Leaves & [58] \\
\hline (+)-2-Hydroxy-I,9,10-trimethoxynoraporphine & B. podagrica & Leaves & \\
\hline
\end{tabular}


Table 3: Chemical constituents isolated from Beilschmiedia species (continued)

\begin{tabular}{|c|c|c|c|}
\hline Compound & Species & Plant part & Ref \\
\hline (+)-2,11-Dihydroxy-1,10-dimethoxyaporphine & B. podagrica & Leaves & \\
\hline \multirow[t]{4}{*}{ Isoboldine } & B. alloiophylla & Bark & [49] \\
\hline & B. elliptica & Bark & [62] \\
\hline & B. podagrica & Bark & [58] \\
\hline & B. tawa & Berries & [61] \\
\hline (-)-Isocaryachine & B. kunstleri & Bark & {$[47]$} \\
\hline Isocorydine & B. podagrica & Leaves & [58] \\
\hline (-)-Kunstleramide & B. kunstleri & Bark & [47] \\
\hline \multirow[t]{2}{*}{ Laurelliptine } & B. elliptica & Bark & [60] \\
\hline & B. podagrica & Bark & [58] \\
\hline \multirow[t]{2}{*}{ (+)-Laurotetanine } & B. kunstleri & Bark & [47] \\
\hline & B. alloiophylla & Bark & [49] \\
\hline Liriodenine & B. alloiophylla & Bark & \\
\hline (S)-3-Methoxynordomesticine & B. alloiophylla & Bark & \\
\hline$(+)-N$-Methylisococlaurine & B. kunstleri & Leaves & [51] \\
\hline O-Methylvelucryptine & B. brevipes & Leaves & [48] \\
\hline (+)-Nornuciferine & B. kunstleri & Bark & [47] \\
\hline Noratherosperminine & B. kunstleri & Bark & \\
\hline Norarmepavine & B. brevipes & Leaves & [48] \\
\hline (+)-Norboldine & B. kunstleri & Leaves & [51] \\
\hline Obscurine & B. obscura & Stem bark & [62] \\
\hline \multirow[t]{2}{*}{ Oreobeiline } & B. alloiophylla & Bark & [49] \\
\hline & B. oreophila & Bark/wood & [63] \\
\hline (-)-Pallidine & B. kunstleri & Leaves & [51] \\
\hline Pipyahyine & B. zenkeri & Stem bark & [16] \\
\hline Secoboldine & B. alloiophylla & Bark & [49] \\
\hline \multirow[t]{2}{*}{ 6-Epioreobeiline } & B. alloiophylla & Bark & \\
\hline & B. oreophila & Bark/wood & [63] \\
\hline Zanthomamide & B. zenkeri & Stem bark & [16] \\
\hline Beilschmin A & B. tsangii & Stem & [50] \\
\hline Beilschmin B & B. tsangii & Stem & \\
\hline Beilschmin C & B. tsangii & Stem & \\
\hline Beilschmin D & B. tsangii & Leaves & [15] \\
\hline Beilschminol A & B. tsangii & Roots & [19] \\
\hline Beilschminol B & B. tsangii & Roots & [45] \\
\hline 4a,5a-Epoxybeilschmin A & B. tsangii & Leaves & [15] \\
\hline 4a,5a-Epoxybeilschmin B & B. tsangii & Leaves & \\
\hline Tsangin A & B. tsangii & Stem & [50] \\
\hline Tsangin B & B. tsangii & Stem & \\
\hline Tsangin $\mathrm{C}$ & B. tsangii & Roots & [19] \\
\hline (+)-Kunstlerone & B. kunstleri & Leaves & {$[47,51]$} \\
\hline Magnolol & B. volckii & Leaves & [14] \\
\hline $\begin{array}{l}\left.\text { rel-(7R,8R,7' } R, 8^{\prime} R\right)-3^{\prime}, 4^{\prime}-\text {-methylenedioxy-3,4,5,5'- } \\
\text { tetramethoxy-7, } 7^{\prime} \text {-epoxylignan }\end{array}$ & B. tsangii & Roots & [19] \\
\hline \multicolumn{3}{|l|}{ 5,5'-dimethoxy-7,7'-epoxylignan } & \\
\hline rel-(7S,8S, $\left.7^{\prime} R, 8^{\prime} R\right)-3,3^{\prime}, 4,4^{\prime}, 5,5^{\prime}-$ & B. tsangii & Roots & \\
\hline hexamethoxylignan & B. tsangii & Stem & {$[50]$} \\
\hline Beilschmieflavonoid A & B. zenkeri & Stem bark & [16] \\
\hline Beilschmieflavonoid B & B. zenkeri & Stem bark & \\
\hline & dimethylpyrano)chalcone & Wood & {$[52]$} \\
\hline 5-Hydroxy-7,8-dimethoxyflavanone & B. zenkeri & Stem bark & [16] \\
\hline Kaempferol 3-rhamnoside & B. miersii & Leaves & [53] \\
\hline Luteolin O-glucoside & B. miersii & Leaves & \\
\hline Quercetin 5-methyl ether & B. miersii & Leaves & \\
\hline Quercetin 3-rhamnoside & B. miersii & Leaves & \\
\hline Quercetin 3-glucuronide & B. miersii & Leaves & \\
\hline $\begin{array}{l}4,2 \text { ', 6'-Trihydroxy-3',4'-methylenedioxo-3- } \\
\text { isopentenylchalcone }\end{array}$ & B. tovarensis & Wood & {$[52]$} \\
\hline$(2 S, 4 R)-5,6,7-$ Trimethoxyflavan-4-ol & B. zenkeri & Stem bark & {$[16]$} \\
\hline$(2 S, 4 R)-4,5,6,7-$ Tetramethoxyflavan & B. zenkeri & Stem bark & \\
\hline \multirow[t]{2}{*}{ Oligandrol } & B. oligandra & Bark & {$[14]$} \\
\hline & B. erythrophloia & Roots & {$[43]$} \\
\hline
\end{tabular}


Table 3: Chemical constituents isolated from Beilschmiedia species (continued)

\begin{tabular}{|c|c|c|c|}
\hline Compound & Species & Plant part & Ref \\
\hline Betulinic acid & B. zenkeri & Stem bark & [16] \\
\hline Sitosterol 3-O- $\beta$-D-glucopyranoside & B. zenkeri & Stem bark & \\
\hline Bisabolene & B. anacardioides & Stem bark & [17] \\
\hline Tricosanoic acid & B. anacardioides & Stem bark & \\
\hline Suberosol B & B. erythrophloia & Roots & [18] \\
\hline $24(S)-3 \beta$-Hydroxystigmast-5-en-7-one & B. erythrophloia & Roots & \\
\hline Lupeol & B. erythrophloia & Roots & \\
\hline 3-O-Acetyl-epi-betulinic acid & B. erythrophloia & Roots & \\
\hline Methyl oleate & B. erythrophloia & Roots & \\
\hline Methyl palmitate & B. erythrophloia & Roots & \\
\hline Methyl linoleate & B. erythrophloia & Roots & \\
\hline \multirow[t]{3}{*}{ 4-Hydroxy-3-methoxybenzaldehyde } & B. erythrophloia & Roots & \\
\hline & B. tsangii & Roots & [19] \\
\hline & B. tsangii & Stem & [50] \\
\hline \multirow[t]{2}{*}{$\alpha$-Tocopheryl quinone } & B. erythrophloia & Roots & \\
\hline & B. tsangii & Stem & [50] \\
\hline \multirow[t]{3}{*}{$6 \beta$-Hydroxystigmast-4-en-3-one } & B. erythrophloia & Roots & [18] \\
\hline & B. tsangii & Roots & [19] \\
\hline & B. tsangii & Stem & [50] \\
\hline 3ß-Hydroxystigmast-5-en-7-one & B. tsangii & Stem & [50] \\
\hline Caryophyllene oxide & B. erythrophloia & Roots & [43] \\
\hline Dehydrooligandrol methyl ether & B. erythrophloia & Roots & \\
\hline Farnesylol & B. erythrophloia & Roots & \\
\hline Oligandrol methyl ether & B. erythrophloia & Roots & \\
\hline \multirow[t]{2}{*}{$\beta$-Sitostenone } & B. erythrophloia & Roots & \\
\hline & B. tsangii & Stem & [50] \\
\hline 3,4,5-Trimethoxybenzaldehyde & B. tsangii & Roots & [45] \\
\hline Eudesm-4(15)-ene-1 $\beta, 6 \alpha$-diol & B. tsangii & Roots & \\
\hline$(4 R, 5 R)-4,5$-Dihydroxycaryophyll-8(13)-ene & B. tsangii & Roots & \\
\hline $\begin{array}{l}\text { Octahydro-4-hydroxy-3a-methyl-7-methylene- } \alpha-(1- \\
\text { methylethyl)-1H-indene-1-methanol }\end{array}$ & B. tsangii & Roots & \\
\hline Ursolic acid & B. tsangii & Roots & \\
\hline (+)-5-Hydroxybarbatenal & B. tsangii & Roots & \\
\hline Sitosterol 3- $\beta$-D-glucopyranoside & B. anacardioides & Stem bark & [46] \\
\hline$\beta$-Amyrone & B. alloiophylla & Bark & [49] \\
\hline 4-Hydroxybenzaldehyde & B. tsangii & Stem & {$[50]$} \\
\hline$\alpha$-Tocospiro B & B. tsangii & Stem & \\
\hline Ergosta-4,6,8(14),22-tetraen-3-one & B. tsangii & Stem & \\
\hline 6a-Hydroxystigmast-4-en-3-one & B. tsangii & Stem & \\
\hline Stigmast-4-en-3,6-dione & B. tsangii & Stem & \\
\hline \multirow[t]{4}{*}{$\beta$-Sitosterol } & B. zenkeri & Stem bark & [16] \\
\hline & B. tsangii & Roots & [19] \\
\hline & B. anacardioides & Stem bark & [44] \\
\hline & B. tsangii & Stem & [50] \\
\hline Taxiphyllin & B. collina & Leaves & [54] \\
\hline
\end{tabular}

\section{BIOLOGICAL ACTIVITIES}

The literature study reveals the need for a thorough investigation of the pharmacological characteristics of the extracts and isolated compounds from Beilschmiedia genus. The biological activities including anti-inflammatory, antioxidant, acetylcholinesterase, anti- $\alpha$ glucosidase, anti-leishmanial, antifungal, antimalarial, anticancer, antitubercular, antiplasmodial, antibacterial and cytotoxicity have been reported in some works. Indeed, the genus Beilschmiedia has been exploited traditionally thus revealed the medicinal variation if possesses. In addition, several species that have been used traditionally to treat some types of ailments have not been investigated for their biological activities at all. Thus, this is an opportunity to find new pharmacological properties from this genus, not to mention promising sources for drugs, such as for cancer, hernia, skin and digestive diseases. Furthermore, the toxicity of the genus members has not been studied from this genus. The information of the qualification of the extracts are very important to be applied as drugs.

\section{Anti-inflammatory activity}

A methanol extract of the roots of $B$. tsangii has shown potent inhibition of NO production, and negative cytotoxicity against RAW 264.7 cells 
[19]. Huang et al [19] also studied the antiinflammatory activity of several compounds isolated from $B$. tsangii. They found that endiandramide A-B exhibited significant iNOS inhibitory activity against RAW 264.7 cells at the concentration range of $0.1-100 \mu \mathrm{M}$. A year later, they reported moderate iNOS inhibitory activity for endiandric acid M. They also suggested that endiandramide $B$ and endiandric acids K-M having the same skeleton but with different substituents at C-8, exhibited ascending degrees of iNOS inhibitory activity in the order: endiandramide $\mathrm{B}(16.40 \mu \mathrm{M})>$ endiandric acid $\mathrm{M}$ $(31.70 \mu \mathrm{M})>$ endiandric acid $\mathrm{L}(39.56 \mu \mathrm{M})>$ endiandric acid $\mathrm{K}(58.21 \mu \mathrm{M})$. This suggests that the iNOS potency of the substituent at C-8 could be arranged as $\mathrm{N}$-isobutylamido group $>$ an $\alpha, \beta$ unsaturated carboxylic acid group $>$ a carboxylic acid group. Endiandric acid M, having four fewer methylenes than endiandric acid L, showed stronger potency than endiandric acid $L$. This also suggests that fewer methylenes in the alkyl side of endiandric acid analogues result in greater potency of iNOS inhibitory activity [45]. In addition, the mixture extracts of the leaves and of $B$. erythrophloia showed weak inhibitory activity of NO production at dose of $25 \mu \mathrm{g} / \mathrm{mL}$ [23].

\section{Antibacterial activity}

B. cinnamomea root extract demonstrated moderate activity against Enterobacter aerogenes EA294 with minimum inhibitory concentration (MIC) value of $64 \mu \mathrm{g} / \mathrm{mL}$ [55]. Three years later, Fankam et al [25] reported on methanol fruits extract of $B$. obscura which showed strong activity against Escherichia coli ATCC8739 with MIC value of $16 \mu \mathrm{g} / \mathrm{mL}$. Besides, another study reported on $B$. tovarensis wood extract displayed significant results against Staphylococcus aureus and Enterococcus faecalis [52]. Anti-bacterial activity on methoxylated flavonoid derivatives from $B$. zenkeri, showed that beilschmieflavonoid B displayed the activity against Streptococcus minor with MIC value of $197.5 \mu \mathrm{M}$. Pipyahyine that was also isolated from this species, showed moderate activity against Bacillus subtilis and Pseudomonas agarici with MIC of 163 and 81.5 $\mu \mathrm{M}$, respectively [16].

Talontsi et al [21] and a co-worker identified cryptobeilic acids A-D, tested for anti-bacterial activity and showed that cryptobeilic acid $A$ gave moderate activity against Escherichia coli (MIC value of $10 \mu \mathrm{M}$ ), while cryptobeilic acid $B$ was active against $P$ seudonamas stutzeri with a MIC value of $10 \mu \mathrm{M}$. In another study, Chouna et al [44] reported that beilschmiedic acid C demonstrated the best activity against Bacillus subtilis and Micrococcus luteus with MIC of 89.5, 5.58 and $11.1 \mu \mathrm{M}$, respectively. Antibacterial activity on endiandric acid derivatives was further studied by Williams et al [20]. It was observed that beilschmiedic acid $\mathrm{A}, \mathrm{K}$ and $\mathrm{L}$ gave moderate activity against clinical isolate of methicillin-resistant Staphylococcus aureus with MIC values of $10-11 \mu \mathrm{M}$.

\section{Cytotoxicity activity}

The in vitro cytotoxicity of the methanol extracts of $B$. erythrophloia showed weak activity against HUVEC (human umbilical vein endothelial cells), MCF-7 (breast cancer cell) line and HL-60 (human promyelocytic leukemia cell) cell lines, showed weak activity with MIC values reaching more than $100 \mu \mathrm{g} / \mathrm{mL}$ at the range of concentration $25-500 \mu \mathrm{g} / \mathrm{mL}$ [23]. In another study, the $B$. acuta fruits, leaves and bark extracts showed the inhibition of CCRF-CEM leukemia cell growth of $65.17,26.35$ and 21.33 $\%$, respectively, as measured by the resazurin reduction assay. In addition, the leaves extract also displayed significant activity towards cell lines HCT116p53 (colon cancer cell), MDA-MB231 (human breast carcinoma cell) and U87MG (human glioblastoma cell) with $\mathrm{IC}_{50}$ of $4.79,6.45$, and $7.46 \mu \mathrm{g} / \mathrm{mL}$, respectively. The bark extract also showed activity against the cell line MDAMB-231 with an $\mathrm{IC}_{50}$ value of $6.66 \mu \mathrm{g} / \mathrm{mL}$ [24].

The in vitro cytotoxicity properties of the leaf oil of $B$. erythrophloia were evaluated against OECM1 (human oral squamous cancer cell), J5 (human hepatocellular carcinoma cell), A549 (human lung adenocarcinoma cell), HT-29 (human colon cancer cell), UACC-62 (human melanoma cell) and K562 (human leukemic cell) and shows $\mathrm{IC}_{50}$ values of $32.6,48.6,38.7,18.9$, 5.8 , and $6.8 \mu \mathrm{g} / \mathrm{mL}$, respectively [39]. Williams et al [20] screened the isolated endiandric acids for their in vitro cytotoxicity activity against $\mathrm{NCl}$ H460 (large cell lung carcinoma), PC-3 (prostate adenocarcinoma) and M14 (amelanotic melanoma cell) lines using a MTT-based assay. They managed to find moderate activity of beilschmiedic acid $\mathrm{L}, \mathrm{I}$ and $\mathrm{K}$ against the $\mathrm{NCl}$ $\mathrm{H} 460$ cell lines with $\mathrm{IC}_{50}$ values of $4.4,5.5$ and $5.9 \mu \mathrm{M}$, respectively. (-)-Kunstleramide was investigated on MTT assays against A375 (human melanoma cell), HT-29 (human colon adenocarcinoma cell), WRL-68 (normal hepatic cell), A549 and PC-3 cells. The results showed moderate activity among all the tested cell lines with $\mathrm{EC}_{50}$ values in the range of $44.74-73.87$ $\mu \mathrm{g} / \mathrm{mL}$ [47]. Chen et al [50] studied the in vitro cytotoxicity of several compounds isolated from $B$. tsangii. They found that tsangin $A-B, \alpha-$ tocospiro $B$ and beilschmin $A$ displayed from 
medium to strong activity against the P-388 (murine leukemia cell) line. In addition, $\alpha$ tocospiro $\mathrm{B}$, exhibited the most cytotoxic against the HT-29 cell line. In addition, six alkaloids isolated from $B$. brevipes were tested for in vitro cytotoxic activity against $\mathrm{P}-388$ cell lines, $\mathrm{O}, \mathrm{O}$ dimethylcoclaurine exhibited significant cytotoxicity with an $\mathrm{IC}_{50}$ of $6.5 \mu \mathrm{g} / \mathrm{mL}$ [48].

\section{Anti-plasmodial activity}

According to Lenta et al [16] among the methoxylated flavonoid tested for in vitro antiplasmodial activity, 5-hydroxy-7,8dimethoxyflavanone exhibited the most significant activity against Plasmodium falciparum chloroquine-resistant strain W2 giving an $\mathrm{IC}_{50}$ value of $9.3 \mu \mathrm{M}$. The other compounds which showed remarkable activity were pipyahyine and betulinic acid which gave $\mathrm{IC}_{50}$ values of 3.7 and $5.2 \mu \mathrm{M}$, respectively. Talontsi et al [21] studied the anti-plasmodial activity of the isolated endiandric acid derivatives from $B$. cryptocaryoides. Cryptobeilic acid B exhibited anti-plasmodial activity against the erythrocytic stages of chloroquine-resistant Plasmodium falciparum strain NF54, followed by tsangibeilin B and cryptobeilic acid $\mathrm{D}$ at a concentration of 10 $\mu \mathrm{g} / \mathrm{mL}$.

\section{Anti-tubercular activity}

Chen et al [15] investigated the in vitro antitubercular effects of the isolates from the leaves of $B$. tsangii against Mycobacterium tuberculosis 90 - 221387. They reported that among the furanoid lignans, beilschmin A-B exhibited potent activity with $I_{50}$ values of 2.5 and $7.5 \mu \mathrm{M}$, respectively. Both compounds also exhibited corresponding epoxy-analogues, 4a,5 $\alpha$ epoxybeilschmin $A-B$ and this revealed that epoxidation of the $\mathrm{C}-4,5$ bond would result in reduced anti-tubercular activity. In another study of anti-tubercular activity, Yang et al [18] verified that erythrophloin $\mathrm{C}$ showed significant activity against Mycobacterium tuberculosis H37Rv (MIC of $50 \mu \mathrm{g} / \mathrm{mL}$ ), comparable to ethambutol (MIC of $6.25 \mu \mathrm{g} / \mathrm{mL})$.

\section{Anti-cancer activity}

The anti-apoptotic proteins $\mathrm{Bcl}-2, \mathrm{Bcl}-\mathrm{xL}, \mathrm{Bcl}-\mathrm{w}$, $\mathrm{Mcl}-1$, and $\mathrm{A} 1$, members of the Bcl-2 family, have become attractive molecular targets for cancer treatment. The discovery of dual inhibitors acting on Bcl-xL and $\mathrm{Mcl}-1$ could play a significant role in cancer treatment. Apart from that, Apel et al [22] had investigated the anti-cancer activity of isolated endiandric acid from $B$. ferruginea, ferrugineic acid $A-K$ against anti-apoptotic proteins $\mathrm{Bcl}-\mathrm{xL}$ and $\mathrm{Mcl}-1$ by using a fluorescence polarization assay. Ferrugineic acid $\mathrm{B}-\mathrm{C}$ and ferrugineic acid $\mathrm{J}$ were proved to have significantly exhibited binding affinity for both anti-apoptotic proteins, Bcl-xL.

Meanwhile, ferrugineic acid D showed only a strong inhibiting activity for anti-apoptotic protein, $\mathrm{Mcl}-1$. Considering the weak binding affinity for Mcl-1 of other tested compounds, it could be postulated that the length of the saturated carbon side chain (preferentially five or seven $\mathrm{CH}_{2}$ groups) associated with a terminal 4hydroxyphenyl ring, play a crucial role for $\mathrm{Bcl}-\mathrm{xL}$ and Mcl-1 binding affinities.

Antioxidant, antifungal, antimalarial, anti- $\alpha-$ glucosidase, acetyl-cholinesterase, and antileishmanial activities

B. erythrophloia extracts showed moderate $\mathrm{DPPH}$ radical scavenging assay with an $\mathrm{EC}_{50}$ value of $13.51 \mu \mathrm{g} / \mathrm{mL}$, comparable to ascorbic acid and quercetin that had $\mathrm{EC}_{50}$ values of 1.5 and $2.3 \mu \mathrm{g} / \mathrm{mL}$, respectively [23]. Mollataghi et al $[47,49]$ have investigated the antioxidant activity by DPPH assay on a new neolignan, (+)kunstlerone and a new dienamide, (-) kunstleramide. They found that (+)-kunstlerone showed strong antioxidant activity with $\mathrm{SC}_{50}$ of $20.0 \mu \mathrm{g} / \mathrm{mL}$, while (-)-kunstleramide exhibited very poor dose-dependent inhibition with $\mathrm{SC}_{50}$ of $179.5 \pm 4.4 \mu \mathrm{g} / \mathrm{mL}$, at the concentration of 200 $25 \mu \mathrm{g} / \mathrm{mL}$. The bark extract of $B$. cinnamomea exhibited moderate in vivo anti-fungal activity with MIC and MFC values in the range of 3.12$6.25 \mathrm{mg} / \mathrm{mL}$, towards six fungal; Candida albicans, Cryptococcus neoformans, Candida tropicali, Candida lusitaniae, Candida guilliermondii and Candida glabrata [26].

Mollataghi et al [49] have reported the anti-fungal activity of the new alkaloid, 2-hydroxy-9methoxyaporphine isolated from $B$. alloiophylla. This compound demonstrated good activity against Candida albicans with MIC value of 8.0 $\mu \mathrm{g} / \mathrm{mL}$. More than a decade ago, Kitagawa et al [34] successfully isolated a bisbenzylisoquinoline alkaloid, (+)-dehatrine from the Indonesian medicinal plant, $B$. madang. The compound was found to exhibit a potent inhibitory activity against the proliferation of the malarial pathogen, Plasmodium falciparum $\mathrm{K} 1$ strain $\left(\mathrm{IC}_{50}\right.$ of 0.17 $\mu \mathrm{M})$, with almost the same activity as quinine $\left(\mathrm{IC}_{50}\right.$ of $\left.0.27 \mu \mathrm{M}\right)$. Mollataghi et al [49] studied the in vitro a-glucosidase activity of the isolated compounds from B. alloiophylla. Three compounds that were found to have moderate activity, were characterized as oreobeiline, 6epioreobeiline and (S)-3-methoxynordomesticine. 
In addition, for cholinesterase and leishmanial activities, the results suggested that 2-hydroxy-9methoxyaporphine, laurotetanine and liriodenine displayed significant acetylcholinesterase (AChE) activity. Meanwhile, the authors deemed that they also possessed significant activity in leishmanial activity.

\section{CONCLUSION}

This compilation includes the traditional uses, phytochemical and pharmacological aspects of the genus Beilschmiedia, and especially provides some analysis of the literature published. Some members of the genus are reported to possess medicinal properties and are used to treat various ailments. Endiandric acid derivatives are major constituents of this genus, and are important chemotaxonomic markers used to identify Beilschmiedia plant species from a phytochemical point of view.

\section{ACKNOWLEDGEMENT}

The authors would like to thank the Ministry of Science, Technology and Innovation, Malaysia (MOSTI), for the financial support under vote Q.J130000.2526.03H93 (GUP) and the Department of Chemistry, Faculty of Science, Universiti Teknologi Malaysia, for research facilities.

\section{REFERENCES}

1. Fouilloy R. Flore du Cameroun, Paris, Muséum National d'Histoire Naturelle 1974; 18: 3-4.

2. Burger W, van der Werff H. Flora costaricensis. Family 80, Lauraceae. Fieldiana. Botany New Series 1990; 23: 1-129.

3. Kostermans AJGH. New Critical Malaysian Plants IV. Reinwardtia 1956; 4: 1-40.

4. Kostermans AJGH. Lauraceae. Reinwardtia 1957a; 4: 193-256.

5. Kostermans AJGH. Lauraceae. Communication of The Forest Reseasch Institute, Indonesia 1957; 57: 1-64.

6. Kostermans AJGH. Miscellaneous Botanical Notes. Reinwardtia 1961; 5: 375-411.

7. Kostermans AJGH. Lauraceae. Reinwardtia 1962; 6: 282-291.

8. Kostermans AJGH. Bibilographia Lauracearum. Department Urusan Research Nasional, P.T. Djulie Archipel, Bogor, 1964.

9. Kostermans AJGH. New Species And Combinations of Lauraceae From Thailand. Nat Hist Bull Siam Soc 1975; 25: 29-44.

10. Kochummen KM. Lauraceae, Tree Flora of Malaya, $A$ Manual for Foresters 4. In F.P.S.Ng, ed. Longman, Kuala Lumpur 1989
11. van der Werff $H$. An annotated key to the genera of Lauraceae in the flora Malesiana region. Blumea 2001; 46: 125-140.

12. van der Werff $H$. A synopsis of genus Beilschmiedia (Lauraceae) in Madagascar. Adansonia 2003; 25: 7792.

13. Iwu MM. Handbook of African Medicinal Plants. CRC Press, Boca Raton, FL, 1993.

14. Banfield JE, Black DC, Collins DJ, Hyland BPM, Lee JJ, Pranowo RS. Constituents of some species of Beilschmiedia and Endiandra (Lauraceae): New endiandric acid and benzopyran derivatives isolated from Beilschmiedia oligandra. Aust J Chem 1994; 47: 587-607.

15. Chen JJ, Chou ET, Peng CF, Chen IS, Yang SZ, Huang HY. Novel epoxyfuranoid lignans and antitubercular constituents from the leaves of Beilschmiedia tsangii. Planta Med 2007; 73: 567-571.

16. Lenta BN, Tantangmo F, Devkota KP, Wansi JD, Chouna $J R$, Soh RCF, Neumann B, Stammler HG, Tsamo E, Sewald N. Bioactive constituents of the stem bark of Beilschmiedia zenkeri. J Nat Prod 2009; 72: 21302134.

17. Chouna JR, Nkeng-Efouet PA, Lenta BN, Wansi JD, Kimbu SF, Sewald N. Endiandric acid derivatives from the stem bark of Beilschmiedia anacardioides. Phytochem Lett 2010; 3: 13-16.

18. Yang PS, Cheng MJ, Peng CF, Chen JJ, Chen IS. Endiandric acid analogues from the roots of Beilschmiedia erythrophloia. J Nat Prod 2009; 72: 5358.

19. Huang YT, Chang HS, Wang GJ, Cheng MJ, Chen $\mathrm{CH}$, Yang YJ, Chen IS. Anti-inflammatory endiandric acid analogues from the roots of Beilschmiedia tsangii. $J$ Nat Prod 2011; 74: 1875-1880.

20. Williams RB, Martin SM, Hu JF, Norman VL, Goering MG, Loss S, O'Neil-Johnson M, Eldridge GR, Starks CM. Cytotoxic and antibacterial Beilschmiedic acids from a Gabonese Species of Beilschmiedia. J Nat Prod 2012; 75: 1319-1325.

21. Talontsi FM, Lamshoft M, Bauer JO, Razakarivony $A A$, Andriamihaja $B$, Strohmann $C$, Spiteller $M$. Antibacterial and antiplasmodial constituents of Beilschmiedia cryptocaryoides. J Nat Prod 2013; 76: 97-102.

22. Apel C, Geny C, Dumontet V, Birlirakis N, Roussi F, Pham VC, Mai HDT, Nguyen VH, Chau VM, Litaudon $M$. Endiandric acid analogues from Beilschmiedia ferruginea as dual inhibitors of $\mathrm{BCl}-\mathrm{xL} / \mathrm{Bak}$ and $\mathrm{Mcl}$ 1/Bid interactions. J Nat Prod 2014; 77: 1430-1437.

23. Lin CT, Chu FH, Tseng YH, Tsai JB, Chang ST, Wang $S Y$. Bioactivity investigation of Lauraceae trees grown in Taiwan. Pharm Biol 2007; 45: 638-644.

24. Kuete V, Tankeo SB, Saeed MEM, Wiench B, Tane $P$, Efferth $T$. Cytotoxicity and modes of action of five Cameroonian medicinal plants against multi-factorial drug resistance of tumor cells. J Ethnopharmacol 2014; 153: 207-219.

Trop J Pharm Res, November 2015; 14(11): 2148 
25. Fankam AG, Kuiate JR, Kuete V. Antibacterial activities of Beilschmiedia obscura and six other Cameroonian medicinal plants against multi-drug resistant Gramnegative phenotypes. BMC Complement Altern Med 2014; 14: 241-250.

26. Dzoyem JP, Tchuenguem RT, Kuiate JR, Teke GN, Kechia FA, Kuete $V$. In vitro and in vivo antifungal activities of selected Cameroonian dietary spices. BMC Complement Altern Med 2014; 14: 58-65.

27. van der Werff $H$, Richter HG. Toward an improved classification of Lauraceae. Ann Miss Bot Gard 1996, 83: 409-418.

28. Nishida S. Revision of Beilschmiedia (Lauraceae) in the Neotropics. Ann Miss Bot Gard 1999; 86 657-701.

29. Burkill IH. A dictionary of economic products of the Malay Peninsula; Ministry of Agriculture and Co-operative: Kuala Lumpur, Malaysia, 1966.

30. Long DG. Notes relating to the flora of Bhutan: VIII Lauraceae. Notes Roy Bot Gard Edinburgh 1984; 41: 505-525.

31. Turner IM. A catalogue of the vascular plants of Malaya. Gardens Bulletin, Singapore 1995; 47: 347-757.

32. Perry LM. Medicinal plants of East and Southeast Asia (Attributed Properties and Uses). The Massachusetts Institute of Technology Press, Cambridge, Massachusetts, London, 1980.

33. Wiart C. Medicinal Plants of Asia and the Pacific. Taylor \& Francis, Boca Raton, London, New York, Vol. 1, 2006.

34. Kitagawa I, Minagawa K, Zhang RS, Hori K, Doi M, Inoue $M$, Ishida $T$, Kimura $M$, Uji T, Shibuya $H$. Dehatrine, an antimalarial bisbenzylisoquinoline alkaloid from the Indonesian medicinal plant Beilschmiedia madang, isolated as a mixture of two rotational isomers. Chem Pharm Bull 1993; 41: 997-999.

35. Kumamoto J, Scora RW. Structure of sarisan, an isomer of myristicin, isolated from the leaf oil of Beilschmiedia miersii. J Agric Food Chem 1970; 18 : 544-545.

36. Scora RW, Scora PE. Essential leaf oil of Persea subgenus, Eriodaphne and closely related Perseoid Genera. J Essent Oil Res 2001; 13: 37-42.

37. Setzer WN, Haber WA. Leaf essential oil composition of five species of Beilschmiedia from Monteverde, Costa Rica. Nat Prod Commun 2007; 2: 79-83.

38. Chaverri C, Ciccio JF. Essential oils from Beilschmiedia pendula (Sw.) Hemsl. (Lauraceae) from Costa Rica. J Essent Oil Res 2010; 22: 259-262.

39. Su YC, Ho CL. Composition and in-vitro cytotoxic activities of the leaf essential oil of Beilschmiedia erythrophloia from Taiwan. Nat Prod Commun 2013, 8: 143-144.

40. Salleh WMNHW, Ahmad F, Yen KH. Chemical compositions and biological activities of the essential oils of Beilschmiedia madang Blume (Lauraceae). Arch Pharm Res 2014; 38: 485-493.

41. Salleh WMNHW, Ahmad F, Yen KH, Zulkifli RM. Chemical composition and biological activities of essential oil of Beilschmiedia pulverulenta. Pharm Biol 2015; in press.

42. Eder $C$, Kogler H, Haag-Richter S. Endiandric acid $H$ and derivatives, procedure for its production and use of the same in the treatment of asthma. German patent 10235624, 2004.

43. Yang PS, Cheng MJ, Chen JJ, Chen IS. Two new endiandric acid analogs: $A$ new benzopyran, and a new benzenoid from the roots of Beilschmiedia erythrophloia. Helvet Chim Acta 2008; 91: 21302138.

44. Chouna JR, Nkeng-Efouet PA, Lenta BN, Devkota KP, Neumann B, Stammler HG, Kimbu SF, Sewald N. Antibacterial endiandric acid derivatives from Beilschmiedia anacardioides. Phytochemistry 2009; 70: 684-688

45. Huang YT, Chang HS, Wang GJ, Lin CH, Chen IS. Secondary metabolites from the roots of Beilschmiedia tsangii and their anti-inflammatory activities. Int J Mol Sci 2012; 13: 16430-16443.

46. Chouna JR, Nkeng-Efouet PA, Lenta BN, Wansi JD, Neumann B, Stammler HG, Kimbu SF, Sewald N. Beilschmiedic acids $F$ and $G$ : Further endiandric acid derivatives from Beilschmiedia anacardioides. Helvet Chim Acta 2011; 94: 1071-1076.

47. Mollataghi A, Hadi AH, Shiau-Chuen C. (+)Kunstleramide, a new antioxidant and cytotoxic dienamide from the bark of Beilschmiedia kunstleri Gamble. Molecules 2012; 17: 4197-4208.

48. Pudjiastuti P, Mukhtar MR, Hadi AH, Saidi N, Morita $H$, Litaudon M, Awang K. (6,7-Dimethoxy-4methylisoquinolinyl)-(4'-methoxyphenyl)-methanone, a new benzylisoquinoline alkaloid from Beilschmiedia brevipes. Molecules 2010; 15: 2339-2346.

49. Mollataghi A, Coudiere E, Hadi AH, Mukhtar MR, Awang $K$, Litaudon M, Ata A. Anti-acetylcholinesterase, anti$\alpha$-glucosidase, antileishmanial and antifungal activities of chemical constituents of Beilschmiedia Species. Fitoterapia 2012; 83: 298-302.

50. Chen JJ, Chou ET, Duh CY, Yang SZ, Chen IS. New cytotoxic tetrahydrofuran and dihydrofuran type lignans from the stem of Beilschmiedia tsangii. Planta Med 2006; 72: 351-357.

51. Mollataghi A, Hadi AH, Awang K, Mohamad J, Litaudon M, Mukhtar MR. (+)-Kunstlerone, a new antioxidant neolignan from the leaves of Beilschmiedia kunstleri Gamble. Molecules 2011; 16: 6582-6590.

52. Suarez LEC, Vargas OEB. New chalcones from Beilschmiedia tovarensis. Rev Columb Quim 2005; 34: 35-41.

53. Harborne JB, Mendez J. Flavonoids of Beilschmiedia miersii. Phytochemistry 1969; 8: 763-764.

54. Miller RE, Tuck KL. Reports on the distribution of aromatic cyanogenic glycosides in Australian tropical rainforest tree species of the Lauraceae and Sapindaceae. Phytochemistry 2013; 92: 146-152.

55. Fankam AG, Kuete V, Voukeng IK, Kuiate JR, Pages JM. Antibacterial activities of selected Cameroonian

Trop J Pharm Res, November 2015; 14(11): 2149 
spices and their synergistic effects with antibiotics against multidrug-resistant phenotypes. BMC Complement Altern Med 2011; 11: 104-114.

56. Tchoula JM. Inventaire Ethnobotanique des plantes medicinales du Noun (Ouest-Cameroun) utilisées pour soigner les infections fongiques et bactériennes, Mémoire de Maitrise de Biologie Végétale, Université de Dschang, 2001.

57. Nkeng-Efouet PA. Phytochemicals from Beilschmiedia anacardioides and their biological significance, phytochemicals - A global perspective of their role in nutrition and health. In Tech. Edited by Venketeshwer R, 2012.

58. Johns SR, Lamberton JA, Sioumis AA, Tweeddale HJ. New aporphine alkaloids from Beilschmiedia podagrica. Aust J Chem 1969; 22: 1277-1281.

59. Kerry-Nicholls $\mathrm{JH}$. The origin, physical characteristics and manners and customs of the Maori race, from data derived during a recent exploration of the King Country. New Zeal J Roy Anthropol Inst Great Brit Ireland 1886; 15: 187-209.

60. Clezy PS, Gellert E, Lau DYK, Nichol AW. The alkaloids of Beilschlmiedia elliptica. Aust J Chem 1966; 19: 135-142.

61. Russell GB, Fraser JG. Identification of isoboldine as the major alkaloid form berries of Beilschmiedia tawa. New Zeal J Sci 1969; 12: 694-695.

62. Lenta BN, Chouna JR, Nkeng-Efouet PA, Fon KS, Tsamo E, Sewald N. Obscurine, a new cyclostachine acid derivative from Beilschmiedia obscura. Nat Prod Commun 2011; 6: 1591-1592.

63. Tillequin F, Koch M, Pusset J, Chauviere G. Two new morphinane alkaloids from Beilschmiedia oreophila Schlechter (Lauraceae). Heterocycles 1985; 23: 1357-1361. 